\title{
Ultrafast Doppler imaging and Ultrasound Localization Microscopy reveal the complexity of vascular rearrangement in chronic spinal lesion
}

\section{Benoit Beliard}

Inserm U1273, ESPCI PSL Paris, CNRS UMR8361, PSL Research University - Paris

\section{Chaimae Ahmanna}

CNRS UMR 8246, INSERM U 1130, Sorbonne Université Sciences, Institut de Biologie Paris Seine IBPS

\section{Elodie Tiran}

Inserm U1273, ESPCI PSL Paris, CNRS UMR8361, PSL Research University - Paris

\section{Kadia Kante}

CNRS UMR 8246, INSERM U 1130, Sorbonne Université Sciences, Institut de Biologie Paris Seine IBPS

\section{Thomas Deffieux}

Inserm U1273, ESPCI PSL Paris, CNRS UMR8361, PSL Research University - Paris

\section{Mickael Tanter}

Inserm U1273, ESPCI PSL Paris, CNRS UMR8361, PSL Research University - Paris

\section{Fatiha Nothias}

CNRS UMR 8246, INSERM U 1130, Sorbonne Université Sciences, Institut de Biologie Paris Seine IBPS

\section{Sylvia Soares}

CNRS UMR 8246, INSERM U 1130, Sorbonne Université Sciences, Institut de Biologie Paris Seine IBPS

\section{Sophie Pezet ( $\square$ sophie.pezet@espci.fr)}

Inserm U1273, ESPCI PSL Paris, CNRS UMR8361, PSL Research University - Paris

\section{Research Article}

Keywords: neuroimaging, blood flow, super-resolution, ultrasound localization microscopy, neuroimaging, spinal cord traumatic injury

Posted Date: January 4th, 2022

DOI: https://doi.org/10.21203/rs.3.rs-1001156/v2

License: (c) (1) This work is licensed under a Creative Commons Attribution 4.0 International License. Read Full License 
Version of Record: A version of this preprint was published at Scientific Reports on April 21st, 2022. See the published version at https://doi.org/10.1038/s41598-022-10250-8. 


\section{Abstract}

Acute spinal cord injury (SCl) leads to severe damage to the microvascular network. The process of spontaneous repair is accompanied by formation of new blood vessels; their functionality, however, presumably very important for functional recovery, has never been clearly established, as most studies so far used fixed tissues. Here, combining ultrafast Doppler imaging and Ultrasound Localization Microscopy (ULM) on the same animals, we proceeded at a detailed analysis of structural and functional vascular alterations associated with the establishment of chronic $\mathrm{SCl}$, both at macroscopic and microscopic scales. Using a standardized animal model of $\mathrm{SCl}$, our results demonstrate striking hemodynamic alterations in several subparts of the spinal cord: a reduced blood velocity in the lesion site, and an asymmetrical hypoperfusion caudal but not rostral to the lesion. In addition, the worsening of many evaluated parameters at later time points suggests that the neoformed vascular network is not yet fully operational, and reveals ULM as an efficient in vivo readout for spinal cord vascular alterations. Finally, we show statistical correlations between the diverse biomarkers of vascular dysfunction and SCI severity. The imaging modality developed here will allow evaluating recovery of vascular function over time in pre-clinical models of $\mathrm{SCl}$. Also, used on SCl patients in combination with other quantitative markers of neural tissue damage, it may help classifying lesion severity and predict possible treatment outcomes in patients.

\section{Introduction}

Traumatic injuries of the spinal cord (SCl) can lead to life-long loss of sensation and voluntary motor functions. Although injured adult neurons of the mammalian central nervous system (CNS) can initially survive, regrowth of their axons through the lesion ultimately fails due to a cascade of cellular and molecular events leaving the affected neural tissue in a permanently altered, regeneration-inhibited state (for review, see ${ }^{1-3}$. Despite clinical advances in rehabilitation and novel treatments based on neuromodulation that improve their quality of life, patients still suffer from the devastating consequences of $\mathrm{SCl}$, aggravated by physiological and psychological complications. Nevertheless, elucidation of the cellular and molecular mechanisms underlying the complexity of $\mathrm{SCl}$, achieved over the last decades, has raised hope for a future development of clinically applicable therapeutic solutions.

A major complication of $\mathrm{SCl}$ pathophysiology is due to injury-associated vascular damage followed by hypoxia, hemorrhage and edema, which accelerate necrosis of the affected neural tissue. The associated breakdown of the blood-spinal cord barrier favors infiltration of blood-derived monocytes/macrophages that, together with activated microglia, spread inflammation beyond the initial lesion site, contributing to secondary expansion of the lesion and increase in neurological deficits (for review, see ${ }^{4,5}$ ). Therefore, it seems important to develop appropriate biomarkers to better assess the vasculature damage, and the limits of its spontaneous restoration, which still remain not well understood.

The initial mechanical injury of the vascularized spinal cord tissue (for review, see ${ }^{6}$ ) provokes, in the acute phase, a dramatic death of endothelial cells, and a decrease of the vessel density. One week post- 
injury, local neo-angiogenesis starts ${ }^{7-11}$, but only part of the newly formed blood vessels become stable and functionally integrated. Therefore, the density of mature vessels remains low in comparison to intact tissue.

Thanks to recent progress in the field of neuroimaging, we now dispose of methods to rapidly and reliably analyze the initial extent of vascular damage on the exposed spinal cord. Thus, using ultrafast Doppler imaging ${ }^{12-15}$ or enhanced ultrasound imaging (involving injection of a contrast agent to enhance sensitivity ${ }^{16,17}$ ), pre-clinical studies have imaged spinal hemodynamics in intact animals ${ }^{15}$, and its hypoperfusion at early stages after $\mathrm{SCl} 12,16,17$.

Combining ultrafast Doppler imaging and Ultrasound Localization Microscopy (ULM) on the same animals, the present study aimed at quantifying trauma-induced alterations of spinal blood volume, main spinal blood flow, density of blood vessels, vessel tortuosity and finally, flow velocity in the various subparts of the vascular network, at two time points post-injury, coinciding with restoration of the bloodspinal cord barrier (4 weeks), and with the establishment of the chronic lesion (8 weeks postlesion) ${ }^{11,18}$. Our study reveals an asymmetric hypoperfusion of the spinal cord associated with reduction of blood flow, alterations of the blood vessel density and morphology, with a worsening at 8-weeks postcontusion.

\section{Results}

\section{Reduced spinal blood volume within the lesion and alterations of the direction of blood flow in lesioned spinal cord}

Imaging the cord's blood volume using ultrafast Doppler imaging (UDI) in a sagittal plane at the level of the midline, with the lesion site being at the center of the imaging plane, allowed for measurements of spinal blood volume (SBV) in different spinal compartments of equivalent surface, at the lesion level, and rostral or caudal to it, in the three groups of animals included in this study. Both at 4- and 8-weeks post-injury, the SBV was strongly reduced at the lesion site, and almost exclusively in the dorsal horn, being non-significant in the ventral horn (Fig. 2C); possibly due to dorso-ventral orientation of the traumatic impact. In addition, although not significant, a tendency of SBV reduction is also noted in caudal segments at both time points post-lesion analyzed, while it was unmodified in rostral segments (Fig. 2A-C).

Using a UDI sequence with a higher sample frequency (Pulse Repetition Frequency $20,000 \mathrm{~Hz}, 5$ compounded plane waves resulting in a $4,000 \mathrm{~Hz}$ frame rate), the blood volumes going toward or away from the probe were separated through spectral analysis, allowing quantification of the main directional blood flow. By convention, a positive value means blood flow in dorso-ventral orientation (Fig. 3A, red arrow), while a flow in the opposite direction has a negative value (blue arrow). 
In intact animals, throughout the whole cord imaged, the main directional blood flow in the dorsal horn is dorso-ventral (Fig. 3A, B red arrow, flow going away from the probe), while in the ventral horn it is a ventrodorsal flow (i.e. going towards the probe, blue arrow, Fig. 3A, B). Four- and eight-weeks post-contusion, the main directional flow along the spinal cord was altered significantly. Both in the dorsal and ventral horns, the main directional flow was reduced (Fig. 3C, D, E) and disorganized (Fig. 3B). Strikingly, in contrast to the measurements of SBV above, the changes of main flow affected the entire cord imaged, not only the lesion site.

\section{Ultrasound localization microscopy for the study of structural vascular abnormalities and alterations in the speed of blood flow}

ULM is capable of localizing and tracking intravenously injected microbubbles, the trajectory of which will then define the vascular arborization. We then used the individual images generated by this analysis (representative examples from the three experimental groups are shown in Fig. 4B) to analyze the anatomical alterations in the blood vessels, such as changes in density (Fig. 4C) and their tortuosity (Fig. 4D), at the lesion site and at rostrally and caudally adjacent segments. Quantification of the density of blood vessels revealed a strong reduction within the lesion that did not change significantly between 4and 8-weeks post-lesion (Fig. 4C, brown frame). This effect is attributable to a reduced blood vessel density in the central sulcar arteries (CSA), the only vascular compartment where the reduction was significant (Fig 4C bottom graphs). Analysis of the tortuosity in these arteries revealed an increase (in tortuosity) with time (Fig. 4D).

Second, we quantified changes in blood velocity in the various vascular compartments of intact / lesioned spinal cords by ULM analysis of microbubble speeds (Fig. 5). As shown in examples in Fig. 5A and quantifications in Fig. 5B, the blood velocities are significantly reduced at 4- and 8-weeks postcontusion compared to intact animals, at the lesion site and caudally to it (Fig. 5B, top panels). Here, both the ASA (anterior spinal artery ) and the CSA are affected only at 8 weeks but not at 4 weeks, suggesting a worsening of this alteration at 8 weeks (Fig. 5B, bottom panels). Accordingly, and as highlighted by the examples in Fig. 5A, one striking difference between 4- and 8-weeks post-contusion is the flow reduction within tortuous blood vessels.

\section{Analysis of vasculature alteration using immunohistochemical staining}

Post-mortem analysis on the same animal was also performed on sagittal spinal cord sections using immunohistochemistry for laminin and for SMI-71, a marker for mature blood vessels ${ }^{8,11}$. While in intact CNS, laminin immuno-labelling reveals basal lamina-associated blood vessels, in traumatic injured SCl, laminin staining was strongly increased at the injury site and in adjacent tissue, revealing the extent of the lesion, as previously described by us and others $8,10,11$ (see Fig. 6A, showing laminin staining from the 
epicenter towards more distal segments,). The density of mature SMI-71 positive blood vessels was strongly reduced at the injury epicenter (Fig. 6B, center panel). When neo-angiogenesis occurred, from 4and 8 weeks post-injury (Fig. 6 A and B), SMI-71 positive vessels were present within, and also around the primary lesion site, which is often replaced by a cavity. However, the organization of newly formed blood vessel network is disorganized (Fig. 6B) and their density remained significantly lower at the lesion site compared to the intact tissue (Fig. 6C).

\section{Definition of new biomarkers of vascular dysfunction associated with spinal cord injury severity}

Finally, in order to decipher potential links between these altered biomedical measures that may be relevant for common neuropathological mechanisms associated with $\mathrm{SCl}$ and help define biomarkers of vascular dysfunction, we studied the statistical correlations between locomotor behavior, SBV and flow, anatomy of blood vessels, and velocity of bubbles, using individual values from all animals included in this study. Results are presented in a double correlation matrix (Fig. 7), in which in Fig. 7A, the Spearman's correlation coefficients are displayed. The statistical relevance of these correlations is shown in Fig. 7B (black/white color code; White: statistically significant, $p<0.05$ - Black: non-significant $(p>0.05)$ ). The corrected $p$-value (correction for multiple comparison using Benjamini-Hochberg test) is indicated in the half top matrix. In both A and B, 6 clusters of statistically significant results were drawn, and Figs. 7C-F present individual values for one example of each cluster.

These analyses show robustly that 1 ) the measure of blood vessel density using ULM correlates very well with the quantification using the 'gold standard', i.e. immunohistochemistry of SMI-71 (pink cluster, Fig. 7C), therefore validating the use of ULM for quantification of blood vessel density. 2) Markers of structural vascular alteration also reveal a significant correlation between the reduced velocity of bubbles and the increased tortuosity of the CSA (purple cluster).

3) The altered locomotor behavior (BBB) is significantly correlated with the reduction of SBV in the dorsal part of the lesion and with the reduced flow along the cord (red cluster). The BBB was also correlated with the velocity. The lower the BBB (higher impairment), the stronger the reduction in bubble velocity (Fig. 7E).

4) The reduction of SBV within the lesion is associated with the anatomical alterations of the blood vessels (reduced density and increased tortuosity, blue and Fig. 7D). Finally, the reduction of bubble velocity is correlated with the reduced flow in the dorsal horn (bright green cluster and Fig. 7F).

\section{Discussion}

Using multimodal fUS imaging and ULM, this study aimed at investigating in depth the anatomical and structural alterations of the vascular arborization at two time points; 4 weeks post-lesion, a phase coinciding with the restoration of the blood-spinal cord barrier and 8 weeks post-lesion with the establishment of the chronic lesion. Our study provides a quantitative study describing the vascular alterations associated with $\mathrm{SCl}$, with a special interest to both macroscale analysis of the blood flow and 
its main orientations, but also at microscopic scale, with a quantification of its density, tortuosity and finally speed of blood flow within these blood vessels. The use of these different parameters provides important missing pieces of the $\mathrm{SCl}$ puzzle and will help, not only to increase our understanding of the vascular pathophysiological mechanisms underlying $\mathrm{SCl}$, but also to define appropriate biomarkers.

\section{Alterations in blood volume and blood flow during the establishment of chronic SCI}

In contrast with conventional ultrasound imaging, ultrafast ultrasound scanners based on plane wave imaging provide a neuroimaging modality extremely sensitive to displacement of particles, such as red blood cells, but also microbubbles, injected intravenously in Ultrasound Localization Microscopy (ULM). Our first goal was to measure the alterations of SBV and main blood flows in the lesioned cord compared to intact animals.

In agreement with previous angiographic observations (reviewed by ${ }^{19}$ ) and more recent, sensitive ultrasound imaging $12,16,17$, our study confirms a strongly decreased SBV in the lesion site, but also demonstrates a lack of SBV alteration in adjacent segments (both rostral and caudal) that was not reported previously. Interestingly, our approach also reveals that this reduced SBV is highly correlated ( $p=$ $10^{-5}$ ) with the individual locomotor disability of the animals, suggesting a link between motor impairment and the amplitude of hypoperfusion. Indeed, it has been reported that the extent of vascular damage is correlated with the development of secondary lesions after $\mathrm{SCl}$, while neo-angiogenesis plays a key role in the progress of functional recovery after $\mathrm{SCl}$, particularly during the chronic injury phase ${ }^{7,20}$. Accordingly, it has been shown recently that promoting angiogenesis and microvessel density after $\mathrm{SCl}$ improves locomotor function recovery ${ }^{20}$.

Furthermore,, analysis of the main directional blood flows, quantified here for the first time,, brought new, interesting results. Whereas the changes of SBV are restricted to the lesion site, the changes in main directional flow are time-dependent, and widespread along the whole thoracic cord, which is eompared to the SBV. Interestingly, unlike changes of SBV, the reduction of top-down flow along the dorsal thoracic cord is linked to the altered vascular morphology in the ventral horn (tortuosity) and the reduced velocity of microbubbles, and these parameters are linked statistically. These results suggest that to assess functional integrity of the spinal blood flow, the measure of the flow directionality is more sensitive than the local measure of SBV. These subtle alterations may be due to the observed anatomical alterations in the arteries (ASA, CSA), (inducing subsequently) leading to a reduced blood flow in the arteries innervating the dorsal horn. 
Previous studies measuring spinal blood volume alterations following $\mathrm{SCl}$ were mainly performed at very early time points (i.e. within hours / days post injury), where the decreased blood volume is due to the initial hemorrhage, followed by spinal ischemia. The grey matter naturally receives the largest blood supply compared to white matter due to its dense network of capillaries. As previously discussed ${ }^{21}$, ischemia in the grey matter therefore leads to a quick and widespread cell death, necrosis, debris formation, rapidly followed by neuroinflammation and cavitation. After the largely documented early decrease in the density of blood vessels ${ }^{7,11,22-26}$, an adaptive vascular response takes place with angiogenesis and re-opening of the microcirculation 7,22,23,27. The time points chosen in our study (4 and 8 weeks post-injury) up to the establishment of the chronic phase, encompass the formation of new blood vessels, but also necrotic cavities. Interestingly, several of our measurements of the spinal structural and functional vasculature integrity (reduced arterial velocity, inverted flow in the ventral horn) showed a worsening between 4 and 8 weeks post-contusion, probably due to the highest progression of secondary lesions leading to cavitation . Indeed, from 4 weeks post-injury on, the immune response becomes a rather persistent inflammatory state. Such environment affects the autonomous tissue repair, including axonal plasticity initiated in the sub-acute phase, but largely aborted in the course of tissue inflammation and necrosis (Milbreta et al., 2014; Chedly et al, 2017; and Soares 2007).

\section{Invaluable contribution of ULM for the estimation of blood vessel density, speed of micro-bubbles and blood vessel density}

in the field of pre-clinical neuroimaging of the lesioned spinal cord, microbubbles were used in the past simply as contrast agents ${ }^{16,17}$. Here, these microbubbles were used differently. We previously demonstrated that in the brain, by imaging at a fast framerate, it is possible to detect individual microbubbles. Thus, microbubbles allowed us to visualize in live animals the fine structure of blood vessels at the microscopic (10 mm) scale, an approach termed 'Ultrasound Localization Microscopy' (ULM) ${ }^{28}$. Tracking of these microbubbles, on the other hand, enables us to measure particle speed, equivalent to local blood velocity, at the same microscopic scale. More recently, we demonstrated that ULM is applicable to the lumbar spinal cord ${ }^{15}$ in intact animals. In the present study, we went one step further, using the invaluable spatial resolution and sensitivity of this technique on lesioned spinal cord to quantify structural damage to the vasculature and changes in blood velocity.

Furthermore, our approach allowed for detailed quantitative measurements of the blood velocity in subparts of the damaged vascularization. The speed of blood flow observed in the lesioned spinal cord is consistent with a previous report by Soubeyrand et al. on early stages post-injury ${ }^{12}$. We convincingly show a massive reduction in blood velocity within and caudal to the lesion at both 4 and 8 weeks postcontusion. Because these changes were also observed in the local arteries (ASA and CSA) that provide $2 / 3$ of the vascularization in the ventral horn ${ }^{29}$, we suggest that the observed effect in the lesion site is due to a reduction in the blood flow in these arteries. As previously quantified using micro-computed 
tomography ${ }^{25,26}$ ), and confirmed here, the shape of the CSA is altered, giving rise to a non-orthogonal ascending flow. The number of branches of the CSA and its diameter are also reduced ${ }^{25,30}$. Our statistical analysis proves that these alterations are correlated with increased tortuosity. It is indeed likely that these structural alterations are the cause for the decreased blood velocity.

Finally, these changes come along with a dramatic reduction of local blood vessel density within and caudal to the lesion at both 4 and 8 weeks post-contusion, as demonstrated both by ULM measurement of the density of blood vessels, and by immunohistochemical quantification of blood vessels in fixed spinal cord of the same animals. Both approaches provided similar, statistically equivalent results, validating the use of ULM for the quantification of structural vascular alterations. Moreover, the observed reduced blood vessel density is consistent with previous reports on hemorrhage and vascular plasticity ${ }^{25}$.

\section{Rostro-caudal asymmetry of the vascular alterations}

So far, only few studies investigated the anatomical and functional damage following SCl by comparing the alterations occurring rostrally versus caudally from the initial lesion site. Strotton et al. ${ }^{21}$, in a thorough spatio-temporal 3D contrast micro-computed tomography (CT) study, elegantly showed the structural alterations in spinal grey and white matters and dorsal columns. They reported that although rostral and caudal adjacent segments undergo similar alterations, their magnitude is significantly higher caudally than in rostral segments. This is particularly true for the vasculature damage (Li et al., 2017), as also demonstrated by our present study. Thus, we found significantly reduced blood velocities in caudal segments compared to intact animals, as well as a tendency for SBV reduction. The pronounced vasculature damage in caudal segments appeared to be related to the unexpected chronic hypoxia in the cord far caudal of the injury epicenter that has recently been described (Li et al., 2017). This study also provided a mechanism that underlies such rostro-caudal asymmetry of vasculature alteration: even months after $\mathrm{SCl}$, the spinal cord below the site of injury remains in a chronic state of hypoxia owing to paradoxical excessive activity of monoamine receptors (5-HT1) on pericytes, despite the absence of monoamines. This monoamine receptor activity causes pericytes to locally constrict capillaries, which reduces blood flow to ischemic levels. Inhibition of monoamine receptors, or increase in inhaled oxygen, produces substantial relief from hypoxia and improves locomotor function recovery. Here, using ULM, our study confirms the strong asymmetry in blood speed between rostral and caudal segments, suggesting that the underlying mechanisms, previously described for 6 months post-lesion (Li et al, 2017), are active much earlier, from the establishment of the chronic lesion on (here shown at 4 and 8 weeks post-injury).

\section{Towards patient's stratification using UDI and ULM}


For SCl pathophysiology reliable prognosis instruments are critically needed, be it for the individualized neurological treatment of patients, or the selection of patients for clinical trials. Based on age and clinical neurological parameters (with or without imaging, depending on the studies), several teams provided prognostic models of the patient's independent walking ${ }^{31,32}$, or urinary continence, one year after $\mathrm{SCl}^{33}$.

In order to go further, the identification and validation of early biomarkers of the degree of neural and vascular damage, predictive of the neurological outcome, is under active investigation. Current biomarkers include imaging readouts of neural alterations, and titrations of particular biomolecules in the cerebrospinal fluid or in the serum of patients (see for review ${ }^{3,34}$ ). The early extent of the hemorrhage and the degree of vascular alteration play a determinant role in the patients' functional recovery. Inclusion of the measurements at a very early stage, i.e. during decompression surgery (when the spinal cord is directly accessible) and possibly later, transcutaneous ${ }^{17}$ if the materials inserted allows ultrasound imaging, would provide accurate information on vascular alterations, including reduced flows in the different spinal vascular compartments.

We previously showed that UDI and ULM are applicable to human brain , both non-invasively in neonates ${ }^{35}$ and adults ${ }^{36}$, and also during perioperative interventions in adult patients ${ }^{37}$. The precise analysis of vasculature state, along with other biomarkers previously described (blood serum cytokines, MRI, DTI ${ }^{38-41}$ ) would provide a more complete picture of the pathophysiological changes in patients with various degrees of injury severity, and allow for a refined/more accurate prognosis in view of the long-term follow up of these patients.

\section{Acknowledgements}

This work was supported by Sorbonne Université and the Institut National de la Santé et de la Recherche Médicale (INSERM), Centre National de Rechercher Scientifique (CNRS), the ANR 'PINCH' (18-CE37-000501), Satt-Lutech (Meduse-MA00444), IRME, Medjeuse, and Inserm ART (Technology Research Accelerator) in "Biomedical Ultrasound".

The authors wish to thank Dr Ysander von Boxberg for careful reading and helpful comments on this manuscript. Finally, three figures contain cartoon drawn using Biorender (app.biorender.com).

\section{Materials And Methods}

All experiments performed in this study were in accordance to the French and European Community Council Directive of September 22 (2010/63/UE). They were also approved by the local Institutional Animal Care and Ethics Committees (\#59, 'Paris Centre et Sud' project \#2018-05 and Sorbonne University project \#1514.01). Accordingly, the number of animals in our study was kept to the minimum necessary. Finally, all methods are in accordance with ARRIVE guidelines. 


\section{Surgical preparation and SCI model}

Animals arrived in the animal facilities at the IBPS institute 2 weeks before the beginning of experiments. Fifty adult female Wistar rats (225-250 g) were obtained from Janvier labs (France) and housed under controlled temperature $\left(22 \pm 1^{\circ} \mathrm{C}\right)$, relative humidity $(55 \pm 10 \%)$ and 12 hours light-dark cycle. Before and after surgical interventions food and water were available ad libitum.

Surgical procedures were performed under reversible, continuous Isoflurane anesthesia $(2 \%-2,5 \%$, Isofluran ${ }^{\circledR}$ ) and sterile precautions were used throughout. In order to perform the spinal cord contusion, skin and musculature was cut from T7-T10 and the dorsal surface of T8-T9 exposed by laminectomy, with the dura remaining intact. The vertebral column was stabilized by fixing vertebral bodies rostrally and caudally to the impact area with clamps attached to the base of the Benchmark ${ }^{\mathrm{TM}}$ impactor device. A $2.5 \mathrm{~mm}$ diameter tip was delivered from $7 \mathrm{~mm}$ height with a speed of $1.96 \mathrm{~m} / \mathrm{s}$ over the spinal cord dorsal surface, and contact time after the impact was $1 \mathrm{~ms}$. These parameters lead to a severe lesion with pronounced locomotor deficits evaluated using the Basso, Beattie and Bresnahan locomotor rating scale (initial BBB score 1 day after the trauma between 0-0.5).

To prevent urinary infections, rats received subcutaneous injections of eurofloxacin (Baytril@ 10\%) once a day during the first week post-lesion. Until restoration of normal micturition, bladders were manually emptied twice a day, and the health state of operated rats monitored by regular weight and visual inspection of the surgical wound.

\section{Fig. 1 Illustration of experimental setup.}

The rats were randomly assigned into three groups, five animals per group: intact (received a sham lesion, where the muscles were opened but the spinal cord left intact), 4- and 8-weeks post-injury. BBB locomotor scores were taken at days 1, 7, and then weekly for a total of 7 weeks. Since the animals were transported to another institute for spinal cord imaging, recording of BBB scores was stopped one week before the experiment. At the end, the rats were sacrificed for histological investigation.

\section{Surgical procedures and preparation for imaging}

Under deep anesthesia (IP) bolus of Medetomidine (Domitor, 0.4 mg.kg-1) and ketamine (Imalgène, 40 mg.kg-1)), a laminectomy (centered on the spinal cord lesion) was performed between thoracic T6 and lumbar L2 vertebrae (two vertebrae above and below the initial laminectomy), thus opening a window allowing positioning of the entire ultrasonic probe $(14 \mathrm{~mm})$ in a sagittal plane.

Thereafter, the animal was placed on a spinal cord stereotaxic frame. Anesthesia was maintained but reduced, using subcutaneous perfusion of Medetomidine $(0.2 \mathrm{mg} / \mathrm{kg} / \mathrm{h})$ and ketamine $(25 \mathrm{mg} / \mathrm{kg} / \mathrm{h})$ 
using a syringe pump. During the surgical procedure and the imaging session, the animal's body temperature was kept at $37^{\circ} \mathrm{C}$ using a heating blanket and an intrarectal probe (Physitemp, USA), and heart and respiratory frequencies were monitored (MouseOxPlus, Ugo Basile, Italy). Each imaging session lasted 2-3 hours.

\section{Ultrafast Doppler imaging and signal analysis}

Two milliliters of saline were gently dropped on the spinal cord (the dura mater was kept intact), and the window created by laminectomy was then filled with echographic gel. The ultrasonic probe $(f=15 \mathrm{MHz}$, $100 \mu \mathrm{m}$ spatial pitch, 128 elements, Vermon, France) was positioned just above the window using a 3axis motor. The probe connected to an ultrasonic ultrafast imager (Iconeus, 128 channels, $62,5 \mathrm{MHz}$ sampling rate) was driven with a prototype software (Iconeus, Paris, France, and Inserm Accelerator of Technological Research in Biomedical Ultrasound, Paris, France). The imaging session started by a 3D scan of the spinal cord that allowed positioning of the probe. By symmetry of the vascular structure, the median plane was deduced and the alignment of the probe with the spinal cord adjusted by rotating it until the anterior spinal artery (ASA) was entirely seen on the images. Ultrafast Doppler at $5,500 \mathrm{~Hz} P R F$ for relative CBV, Ultrafast Doppler at 20,000 Hz PRF for blood flow direction and at 5,000 Hz PRF for ultrasound localization microscopy acquisitions were performed successively.

\section{Measure of SBV using Power Doppler imaging}

SBV values were obtained with the ultrafast Doppler imaging method, which consists of compounded plane-wave ultrasound transmissions ${ }^{42}$. Thus, each frame was a compound plane wave frame resulting from the coherent summation of 11 compounded tilted plane waves with angles separated by $2^{\circ}$ and ranging from $-10^{\circ}$ to $10^{\circ}$. The framerate used was $500 \mathrm{~Hz}$, resulting in a $5,500 \mathrm{~Hz}$ pulse repetition frequency (PRF). The Singular Value Decomposition (SVD) clutter filtering (Demene et al 2015) typically used for brain tissue had to be modified for our use on spinal cord tissue. The SVD thresholds were automatically determined thanks to adaptive spatiotemporal SVD using the similarity of spatial singular vectors ${ }^{43}$. Over 400 images, the first 30 singular values were excluded to remove the tissue and the last 230 were excluded to remove the noise. The resulting 400 successive images were averaged to obtain a single SBV image that was analyzed by spatial averaging of the different ROI drawn using MATLAB 2020a.

Choice and size of areas taken for the measurements of SVB, directions of flow, density of blood vessels and bubble velocity 
- For the quantification (of the SBV, density of blood vessels and bubble velocity) in the lesion, rostral and caudal to the lesion: three subparts of equivalent area (30 mm along the antero-posterior axis $X$ 18-20 $\mathrm{mm}$ dorsal to caudal, i.e. $530 \mathrm{~mm}^{2}$ ) of the thoracic spinal cord were drawn: at the lesion site (around the lesion epicenter), rostral or caudal to it. In intact animals, the same areas were taken, the area at the center was taken for the counter part of the lesion side, and, as in lesioned animals, the same $530 \mathrm{~mm}^{2}$ rostral and caudal to the center were taken for the rostral and caudal values. The respective values were computed on MATLAB.

- Quantifications of bubble velocity in the 'whole cord' (Figure 5), an equivalent rectangle surrounding the entire thoracic cord imaged (or most of it) of the following dimensions $90 \mathrm{~mm}$ (Rostro-caudal) $X$ 18-20 mm (dorso-ventral axis) was computed on MATLAB in all animals included in our study.

- The same principle was used for all the other measures: an equivalent area was defined in all groups, surrounding either the dorsal/ventral thoracic cord (Figure 3), CSA, ASA, dorsal vein (Figs 4 and 5).

\section{Direction of flow}

A different acquisition sequence was used to analyze the direction of flow. Each frame was a compound image of 5 angles $\left(-4^{\circ},-2^{\circ}, 0^{\circ}, 2^{\circ}\right.$ and $\left.4^{\circ}\right)$, which together with a sampling frequency increased to $4000 \mathrm{~Hz}$ resulted in a $20,000 \mathrm{~Hz}$ PRF. The total acquisition contained 2000 frames and lasted 0.5 second. The same SVD filter as for the Power Doppler acquisition was used to separate the tissue signal from the blood signal. Using the same spectral analysis as in the color Doppler images ${ }^{44}$, the SBV signal was separated into two series of images representing the SBV going toward and away from the probe. After averaging the 2000 frames of the two directional SBV, the general directional SBV was obtained by subtracting the SBV going upward from the SBV going downward. The images were then spatially averaged in the same ROIs drawn for the analysis of the SBV previously described.

\section{Ultrasound Localization Microscopy (ULM)}

- A catheter filled with saline was inserted in the rat jugular vein before positioning the animal on the stereotaxic frame. After all previously mentioned measures, ULM was performed similarly to the methods described in ${ }^{36}$ using the same ultrasound imager as above. Compounded frames were acquired at a $1000 \mathrm{~Hz}$ framerate (with angles at $-4^{\circ},-2^{\circ}, 0^{\circ},+2^{\circ},+4^{\circ}, \mathrm{PRF}=5000 \mathrm{~Hz}$ ) using the same system (Iconeus One, Iconeus, Paris, France) as above for a total time of 150s. Beamformed data were filtered using the SVD spatio-temporal filter described in ${ }^{45}$, and the 7 first singular values were removed to extract microbubble signals from the surrounding tissues. Microbubbles were detected as the brightest local maxima in the images. Tracking of the maxima positions was performed using the Iconeus software (Iconeus, Paris, France, and Inserm Accelerator of Technological Research in 
Biomedical Ultrasound, Paris, France) and gives all positions in space (x-axis and z-axis positions) and time (frame number) for each detected individual bubble.

The successive positions gathered in one track were used to compute the interframe bubble velocity vector components (along probe $x$-axis and depth z-axis), and absolute velocity magnitude.

- Density maps were computed by counting all the microbubbles that passed through each pixel during acquisitions. Since the total number of bubbles injected is not exactly the same from one injection to another, comparison of raw density between animals would reflect this random effect instead of intrinsic differences between groups. To avoid this variability, the value of density was normalized by the total number of bubbles detected in the ROI contouring the whole spinal cord. To compare these maps between animals and consider the variability coming from the random number of bubbles injected into the blood stream, the value of density was normalized by the total number of bubbles detected in the spinal cord imaged.

- Analysis of the density of blood vessels using the ULM analysis was performed using Image J. The images obtained at the previous steps were first transformed as black and white pictures. Using a common threshold (131) for all animals, the percentage of staining occupied by the blood vessels was measured in each animal in the six following compartments: Lesion site, rostral or caudal to the lesion site, the ASA or the CSA at the level of the lesion site. For each compartment, the area measured for all animals was similar. Results are expressed as percentage of area occupied by the staining.

- The vessel tortuosity index was computed thanks to the MATLAB function "vessel_tortuosity_index" written by Maz M. Khansari ${ }^{46}$. For each animal, three vessels located rostral, caudal and the last one close to the lesion, were chosen on the density map given by the ULM algorithm. A line was drawn over each one to use it as an input for the function. These values were averaged to obtain the tortuosity index for each animal.

- Each pixel of the velocity maps was computed as the mean velocity of every micro-bubble detected in this pixel during the whole acquisition. These two maps were then spatially averaged in ROls drawn the same way as for the SBV analysis.

\section{Statistical analysis}

For each type of statistical comparison, the same protocol of hypothesis verification was applied to choose the appropriate statistical test. First, the two assumptions needed for parametric tests were tested. The normality of the data distribution of each group was tested with a Lilliefors test and the equality of the variances was tested with a Bartlett's test.

Then, an ANOVA was performed to determine if a difference between two groups could be detected with a statistical test. If one of the two conditions for the parametric tests was not met, the Kruskal-Wallis test 
was performed for the same purpose. If the ANOVA detected a statistically significant difference, pair wise tests were used to determined which groups differ. For data suited for parametric test, a student test was used. A Wilcoxon rank sum test was used otherwise.

In Figure 7, for the double correlation matrix: Considering that some measures did not have a gaussian distribution (and were therefore not compatible with a parametric test), all correlations were computed using Spearman coefficients, (which is the non-parametric counterpart of the Pearson's Linear Correlation Coefficient). The p-values corresponds to the probability of rejection of the null hypothesis that the correlation is equal to zero.

\section{Perfusion and immunohistochemistry}

At the end of the experiment, rats were administered a lethal dose of pentobarbital (Euthasol® $400 \mathrm{mg} / \mathrm{mL} ; 150 \mathrm{mg} / \mathrm{Kg}$ ), and were transcardially perfused with saline $\mathrm{NaCl} 9 \%$ at $37^{\circ} \mathrm{C}$, then with $4 \%$ paraformaldehyde (PFA) in $0.1 \mathrm{M}$ phosphate buffer. PFA-fixed tissues were incubated in $15 \%$ and $30 \%$ sucrose for $24 \mathrm{~h}$, and $48 \mathrm{~h}$ at $4{ }^{\circ} \mathrm{C}$, respectively, then embedded in optimal cutting temperature compound (OCT; Tissue-Tek), quickly frozen in a dry ice/isopentane bath and stored at $-80^{\circ} \mathrm{C} .40 \mu \mathrm{m}$ sagittal sections were cut on a cryostat (Leica CM3050 S) and mounted on glass slides (Superfrost@ Plus).

For immunolabeling, sections were permeabilized with 0,3\% Triton X_-100 in PBS during 5 min at room temperature, and incubated for $1 \mathrm{~h}$ in $10 \%$ bovine serum albumin (BSA)/PBS. Blocking was followed by incubation overnight at room temperature with the primary antibodies diluted in $5 \%$ BSA/PBS. The following primary antibodies were used: rabbit glial fibrillary acidic protein (GFAP; Dako, 1:2000), mouseSMI 71 (Covance, 1:100), rabbit-a-laminin (Sigma, 1:100). Finally, appropriate secondary antibodies (Alexa Fluor 488 and 555 conjugated, 1:1000; Life technologies) diluted in BSA 5\% were used.

Figures showing large longitudinal spinal cord sections were produced using a Zeiss Axio Zoom V16 microscope with image stitching and were processed using ImageJ (NIH, USA).

Quantification of SMI-71 positive cells:

Pictures of 4 sections / rat $(160 \mu \mathrm{m}$ between each section) stained with anti-SMI 71 antibody were taken for quantification. With Image J, a rectangle of $1.6 \mathrm{~mm}^{2}$ was drawn at the level of lesion, as well as at the level of the caudal and rostral part of each section. After fixing the same threshold for each section, particle analysis was performed and the mean value per rat was calculated and compared as total area between each group (intact -uninjured, $4 \mathrm{w}$ and $8 \mathrm{w}$ post-injury; 3 rats per group). One-way Anova was done to compare values for each region between groups.

\section{References}


1. David, S. \& Lacroix, S. Molecular approaches to spinal cord repair. Annu Rev Neurosci 26, 411-440 (2003).

2. Sandvig, A., Berry, M., Barrett, L. B., Butt, A. \& Logan, A. Myelin-, reactive glia-, and scar-derived CNS axon growth inhibitors: expression, receptor signaling, and correlation with axon regeneration. Glia 46, 225-251 (2004).

3. Ahuja, C. S. et al. Traumatic spinal cord injury. Nat Rev Dis Primers 3, 17018 (2017).

4. Fawcett, J. W., Schwab, M. E., Montani, L., Brazda, N. \& Müller, H. W. Defeating inhibition of regeneration by scar and myelin components. Handb Clin Neurol 109, 503-522 (2012).

5. Silver, J., Schwab, M. E. \& Popovich, P. G. Central Nervous System Regenerative Failure: Role of Oligodendrocytes, Astrocytes, and Microglia. Cold Spring Harb Perspect Biol 7, a020602 (2015).

6. Mazensky, D., Flesarova, S. \& Sulla, I. Arterial Blood Supply to the Spinal Cord in Animal Models of Spinal Cord Injury. A Review. Anat. Rec. 300, 2091-2106 (2017).

7. Casella, G. T. B., Marcillo, A., Bunge, M. B. \& Wood, P. M. New Vascular Tissue Rapidly Replaces Neural Parenchyma and Vessels Destroyed by a Contusion Injury to the Rat Spinal Cord. Experimental Neurology 173, 63-76 (2002).

8. Loy, D. N. et al. Temporal progression of angiogenesis and basal lamina deposition after contusive spinal cord injury in the adult rat. J Comp Neuro/ 445, 308-324 (2002).

9. Whetstone, W. D., Hsu, J.-Y. C., Eisenberg, M., Werb, Z. \& Noble-Haeusslein, L. J. Blood-spinal cord barrier after spinal cord injury: Relation to revascularization and wound healing. Journal of Neuroscience Research 74, 227-239 (2003).

10. Benton, R. L., Maddie, M. A., Minnillo, D. R., Hagg, T. \& Whittemore, S. R. Griffonia simplicifolia isolectin B4 identifies a specific subpopulation of angiogenic blood vessels following contusive spinal cord injury in the adult mouse. J Comp Neuro/ 507, 1031-1052 (2008).

11. Milbreta, U., von Boxberg, Y., Mailly, P., Nothias, F. \& Soares, S. Astrocytic and Vascular Remodeling in the Injured Adult Rat Spinal Cord after Chondroitinase ABC Treatment. Journal of Neurotrauma 31, 803-818 (2014).

12. Soubeyrand, M., Badner, A., Vawda, R., Chung, Y. S. \& Fehlings, M. G. Very High Resolution Ultrasound Imaging for Real-Time Quantitative Visualization of Vascular Disruption after Spinal Cord Injury. J Neurotrauma 31, 1767-1775 (2014).

13. Song, P. et al. Functional Ultrasound Imaging of Spinal Cord Hemodynamic Responses to Epidural Electrical Stimulation: A Feasibility Study. Front Neuro/ 10, 279 (2019). 
14. Tang, S. et al. Changes in spinal cord hemodynamics reflect modulation of spinal network with different parameters of epidural stimulation. Neurolmage 221, 117183 (2020).

15. Claron, J. et al. Large-scale functional ultrasound imaging of the spinal cord reveals in-depth spatiotemporal responses of spinal nociceptive circuits in both normal and inflammatory states. Pain 162, 1047-1059 (2021).

16. Khaing, Z. Z. et al. Contrast-enhanced ultrasound to visualize hemodynamic changes after rodent spinal cord injury. Journal of Neurosurgery: Spine 29, 306-313 (2018).

17. Khaing, Z. Z. et al. Transcutaneous contrast-enhanced ultrasound imaging of the posttraumatic spinal cord. Spinal Cord 58, 695-704 (2020).

18. James, N. D. et al. Conduction Failure following Spinal Cord Injury: Functional and Anatomical Changes from Acute to Chronic Stages. Journal of Neuroscience 31, 18543-18555 (2011).

19. Tator, C. H. \& Fehlings, M. G. Review of the secondary injury theory of acute spinal cord trauma with emphasis on vascular mechanisms. Journal of Neurosurgery 75, 15-26 (1991).

20. Cheng, X., Xu, J., Yu, Z., Xu, J. \& Long, H. LncRNA Xist Contributes to Endogenous Neurological Repair After Chronic Compressive Spinal Cord Injury by Promoting Angiogenesis Through the miR-325p/Notch-1 Axis. Frontiers in Cell and Developmental Biology 8, 744 (2020).

21. Strotton, M. C. et al. The spatiotemporal spread of cervical spinal cord contusion injury pathology revealed by 3D in-line phase contrast synchrotron X-ray microtomography. Experimental Neurology 336, 113529 (2021).

22. Imperato-Kalmar, E. L., McKinney, R. A., Schnell, L., Rubin, B. P. \& Schwab, M. E. Local Changes in Vascular Architecture Following Partial Spinal Cord Lesion in the Rat. Experimental Neurology 145, 322328 (1997).

23. Zhang, Z. \& Guth, L. Experimental Spinal Cord Injury: Wallerian Degeneration in the Dorsal Column Is Followed by Revascularization, Glial Proliferation, and Nerve Regeneration. Experimental Neurology 147, 159-171 (1997).

24. Hu, J., Cao, Y., Wu, T. \& Li, D. High-resolution three-dimensional visualization of the rat spinal cord microvasculature by synchrotron radiation micro-CT. 101904, (2014).

25. Jiang, L. et al. SR $\mathrm{CCT}$ Reveals 3D Microstructural Alterations of the Vascular and Neuronal Network in a Rat Model of Chronic Compressive Thoracic Spinal Cord Injury. Aging and disease 11, 603 (2020).

26. Cao, Y., Wu, T., Li, D., Ni, S. \& Hu, J. Three-dimensional imaging of microvasculature in the rat spinal cord following injury. Scientific Reports 29, 1-11 (2015). 
27. Blight, A. R. Morphometric analysis of blood vessels in chronic experimental spinal cord injury: Hypervascularity and recovery of function. Journal of the Neurological Sciences 106, 158-174 (1991).

28. Errico, C. et al. Ultrafast ultrasound localization microscopy for deep super-resolution vascular imaging. Nature 527, (2015).

29. Hu, J.-Z. et al. Three-dimensional alteration of microvasculature in a rat model of traumatic spinal cord injury. J Neurosci Methods 204, 150-158 (2012).

30. Ni, S. et al. Synchrotron Radiation Imaging Reveals the Role of Estrogen in Promoting Angiogenesis After Acute Spinal Cord Injury in Rats. Spine (Phila Pa 1976) 43, 1241-1249 (2018).

31. van Middendorp, J. et al. A clinical prediction rule for ambulation outcomes after traumatic spinal cord injury: a longitudinal cohort study. Lancet (London, England) 377, (2011).

32. Wilson, J. R. et al. A Clinical Prediction Model for Long-Term Functional Outcome after Traumatic Spinal Cord Injury Based on Acute Clinical and Imaging Factors. Journal of Neurotrauma 29, 2263-2271 (2012).

33. Pavese, C. et al. Prediction of Bladder Outcomes after Traumatic Spinal Cord Injury: A Longitudinal Cohort Study. PLoS Med 13, e1002041 (2016).

34. Badhiwala, J. H., Ahuja, C. S. \& Fehlings, M. G. Time is spine: a review of translational advances in spinal cord injury: JNSPG 75th Anniversary Invited Review Article. Journal of Neurosurgery: Spine 30, 118 (2019).

35. Demene, C. et al. Functional ultrasound imaging of brain activity in human newborns. Science Translational Medicine $\mathbf{9}$, (2017).

36. Demené, C. et al. Transcranial ultrafast ultrasound localization microscopy of brain vasculature in patients. Nature Biomedical Engineering 5, 219-228 (2021).

37. Imbault, M., Chauvet, D., Gennisson, J.-L., Capelle, L. \& Tanter, M. Intraoperative Functional Ultrasound Imaging of Human Brain Activity. Sci Rep 7, 7304 (2017).

38. Wang, K. et al. Compared study of routine magnetic resonance imaging and diffusion tensor tractography on the predictive value of diagnosis and prognosis in acute cervical spinal cord injury. Journal of Acute Disease 5, 328-332 (2016).

39. D'souza, M. M., Choudhary, A., Poonia, M., Kumar, P. \& Khushu, S. Diffusion tensor MR imaging in spinal cord injury. Injury 48, 880-884 (2017).

40. Matsushita, A. et al. Can the acute magnetic resonance imaging features reflect neurologic prognosis in patients with cervical spinal cord injury? The Spine Journa/ 17, 1319-1324 (2017). 
41. Ogurcov, S. et al. Blood Serum Cytokines in Patients with Subacute Spinal Cord Injury: A Pilot Study to Search for Biomarkers of Injury Severity. Brain Sciences 11, 322 (2021).

42. Montaldo, G., Tanter, M., Bercoff, J., Benech, N. \& Fink, M. Coherent plane-wave compounding for very high frame rate ultrasonography and transient elastography. IEEE Transactions on Ultrasonics, Ferroelectrics, and Frequency Control (2009) doi:10.1109/TUFFC.2009.1067.

43. Baranger, J. et al. Adaptive Spatiotemporal SVD Clutter Filtering for Ultrafast Doppler Imaging Using Similarity of Spatial Singular Vectors. IEEE Transactions on Medical Imaging 37, 1574-1586 (2018).

44. Mace, E. et al. Functional ultrasound imaging of the brain: theory and basic principles. IEEE Trans. Ultrason., Ferroelec., Freq. Contr. 60, 492-506 (2013).

45. Demené, C. et al. Spatiotemporal Clutter Filtering of Ultrafast Ultrasound Data Highly Increases Doppler and fUltrasound Sensitivity. IEEE Transactions on Medical Imaging 34, 2271-2285 (2015).

46. Khansari, M. M. et al. Automated fine structure image analysis method for discrimination of diabetic retinopathy stage using conjunctival microvasculature images. Biomed Opt Express 7, 25972606 (2016).

\section{Declarations}

\section{Conflict of interest}

MT and TD are co-founders and shareholders of Iconeus company. MT is co-inventor of several patents in the field of neurofunctional ultrasound and ultrafast ultrasound. MT and TD do not have any other financial conflict of interest, nor any non-financial conflict of interests. All the other authors do not have any financial or non-financial conflict of interests.

\section{Authors contribution statement}

SP, FN and SS designed the experimental paradigm and wrote the manuscript.

$\mathrm{BB}, \mathrm{CA}, \mathrm{ET}, \mathrm{KK}, \mathrm{FN}, \mathrm{SS}$ and SP performed the experiments.

BB and ET analyzed the ultrasound / ULM data.

MT and TD supervised the signal processing of the ultrasound / ULM data.

$\mathrm{CA}, \mathrm{KK}, \mathrm{FN}$ and SS performed the anatomical / immunohistochemical study and its quantification.

All authors were involved in the interpretation of the data and wrote some parts of the manuscript. 


\section{Figures}

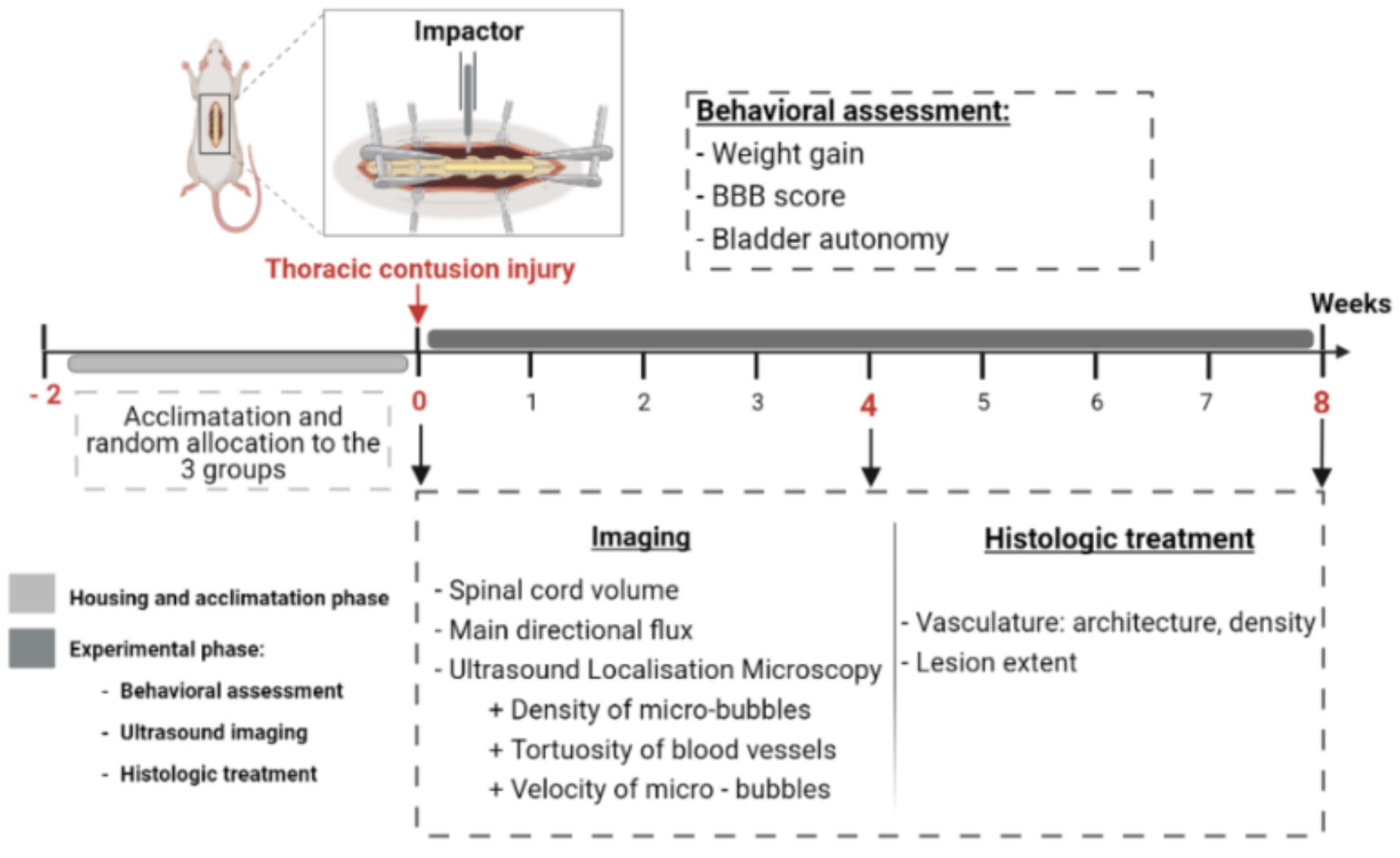

Figure 1: Schematic outline of the experimental design. $\mathrm{N}=10$ animals received a contusion of the thoracic spinal cord (thoracic levels T8-T9). A behavioral assessment was performed before and once a week after the lesion. At 4 and 8 weeks after contusion, spinal cords of $\mathrm{N}=5$ animals per group were imaged through an opening created by laminectomy. At the end of the imaging session, animals were cardially perfused in order to perform immunohistological analyses.

\section{Figure 1}

See figure for legend. 

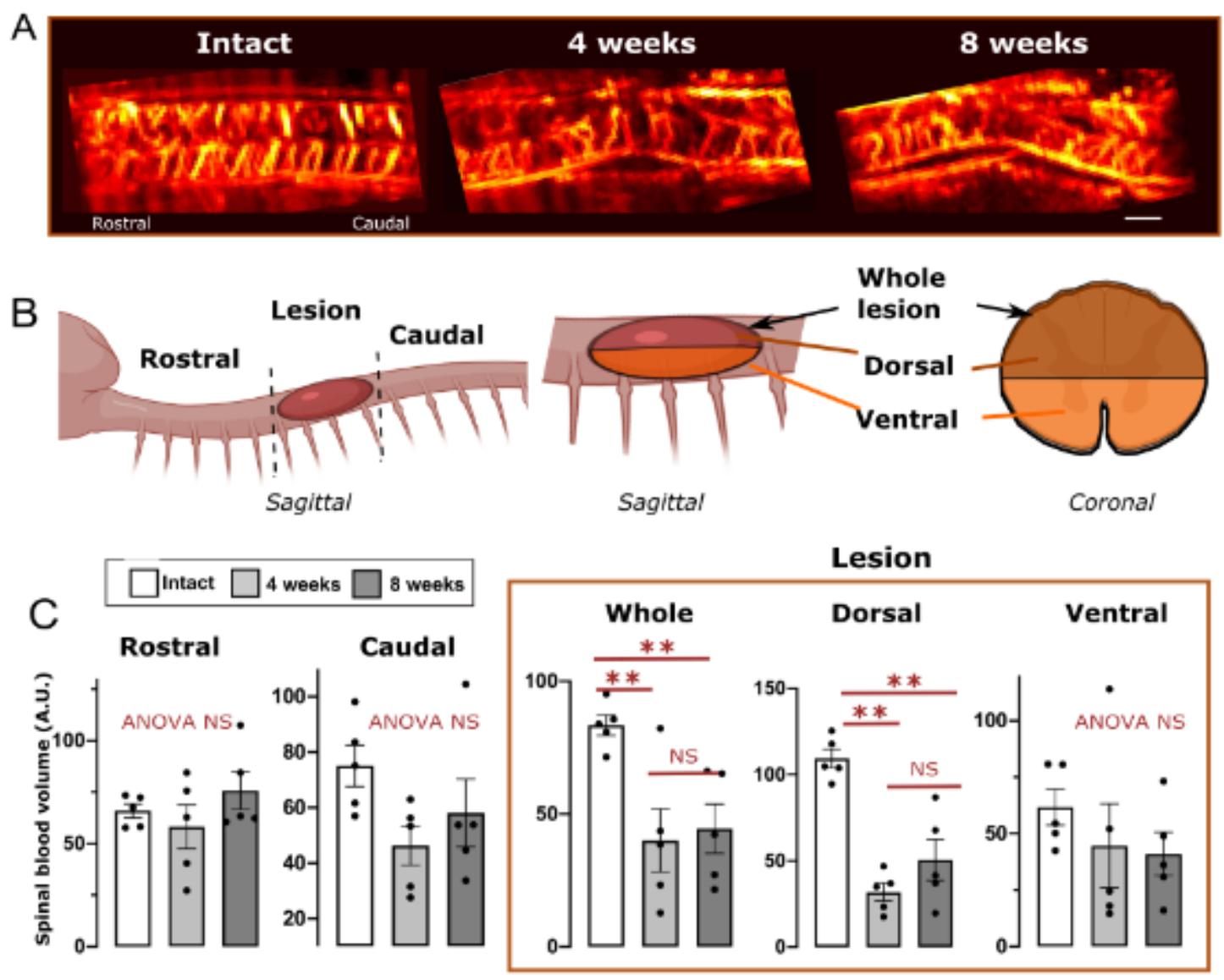

Figure 2: Spinal cord contusion leads to a reduction in spinal blood volume (SBV) in different parts of the lesioned spinal cord at 4- and 8-weeks post-contusion. A: Examples of fUS Doppler images in representative animals. Imaging was performed in the sagittal plane at the level of the midline. B: Cartoons representing the location of SBV analysis rostral or caudal to the lesion, or within the dorsal or ventral part of the lesion site. C: Quantifications show no significant changes in SBV rostral or caudal to the lesion. A clear decrease of SBV is found at the lesion site, attributable exclusively to the dorsal horn. Results are expressed as mean spinal blood volume (Arbitrary units (A.U.)) \pm SEM, overlaid with individual values. $N=5$ animals per groups. Stats: ANOVA, followed by unpaired t-test as post-hoc test. ${ }^{*} p<0.05, * * p<0.01$. NS: Not statistically significant, ANOVA NS:

ANOVA $p>0.05$. Bar : $1 \mathrm{~mm}$.

Figure 2

See figure for legend. 

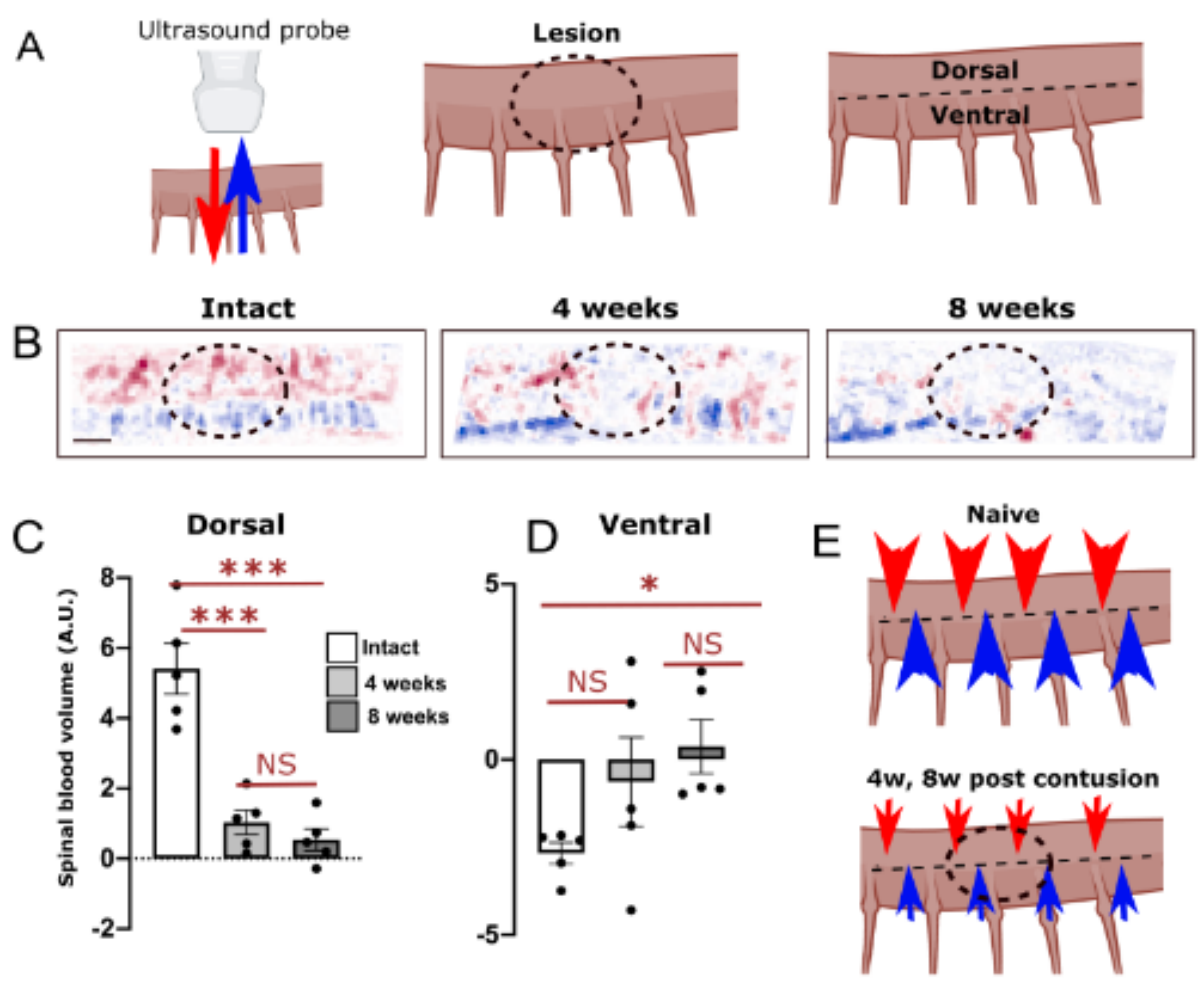

Figure 3: Alterations of mean directional spinal blood volumes over the whole cord 4- and 8-weeks post-contusion. Using a fast sequence of imaging, the main directional flow in the whole dorsal or ventral part of the thoracic cord was quantified. A: Cartoons illustrating the mean directional blood flow (left), the localization of the lesion in the images displayed in B (middle), and the separation between the dorsal and ventral parts analyzed here (right panel). By convention, a positive flow is directed away from the probe, i.e. dorso-ventrally oriented (A, left panel, red arrow). B: Representative examples of directional spinal blood volumes in animals of the three groups studied. In intact animals, the main directional blood volume is upward in the ventral horn and downwards in the dorsal horn. At both 4 and 8 weeks post-contusion, these flows are deeply altered. Quantification of these changes (C, D) show a statistically significant decreased directional flow over the whole dorsal horn (C) and a more modest reduction in the ventral horn (D). E: Schematic of the results obtained, highlighting the fact that the changes are widespread in the hemicords. Results in C-D are expressed as mean spinal blood volume (A.U.) \pm SEM and are presented in overlay with individual values. $\mathrm{N}=5$ animals per groups. Stats: ANOVA, followed by unpaired t-test as post-hoc test. $* \mathrm{p}<0.05, * * *$ $\mathrm{p}<0.001$. NS: Not statistically significant. Bar : $1 \mathrm{~mm}$.

Figure 3

See figure for legend. 

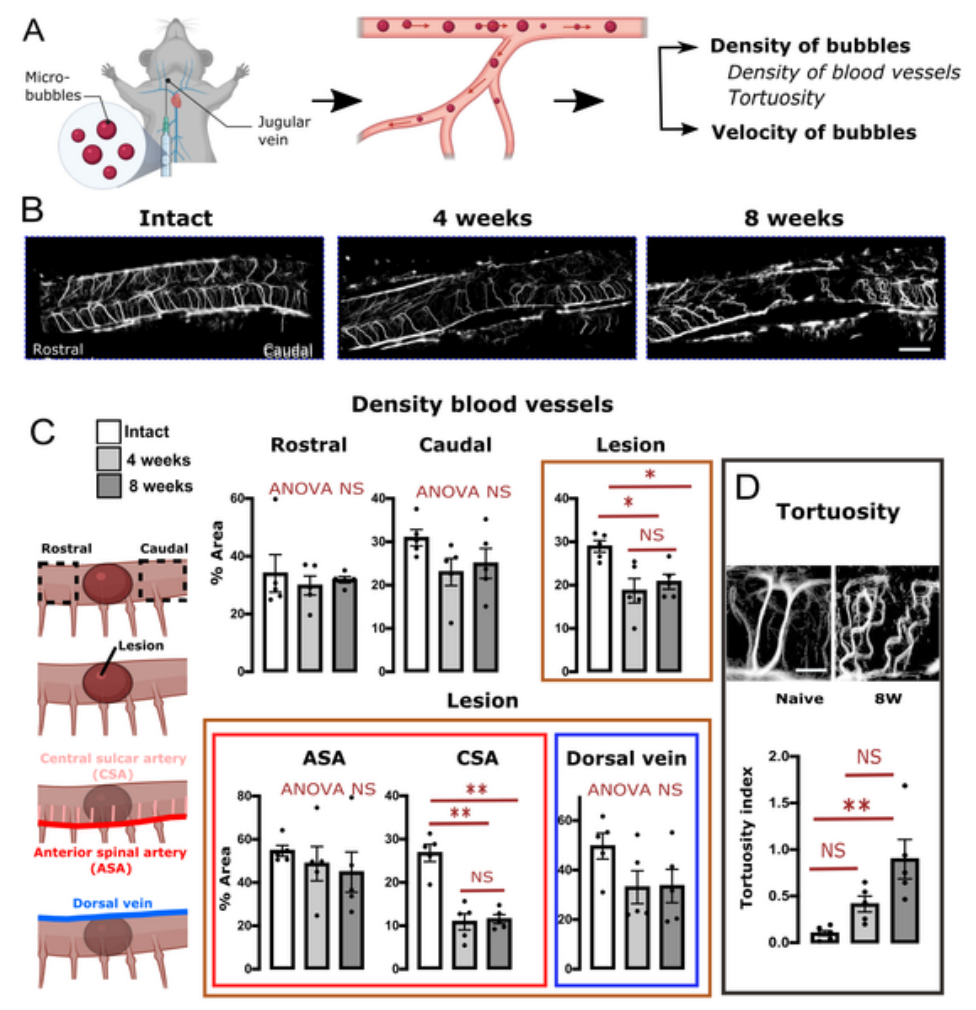

Figure 4: Blood vessel density and tortuosity changes after spinal cord contusion revealed by ULM. A: Schematic of ULM. At the end of the above fUS imaging, 150 ? I of contrast agent (bio-compatible microbubbles) were injected intravenously. Using a fast sequence of imaging and further single bubble localization and tracking, this approach allows to determine the normalized density of bubbles and the velocity of the bubbles (which is a proxy for the local velocity of blood flow). B: Representative examples of images of density of microbubbles in intact animals, and 4- and 8- weeks post-contusion. C: Quantification of the density of blood vessels in various compartments of the lesioned spinal cord (performed using images of the normalized density of bubbles) reveals loss of blood vessels within the lesion and in central sulcar arteries (CSA). D:

Quantification of the tortuosity of the central sulcar artery, using ULM. Top panel: High power magnification images of a intact animal (right) and a lesioned animal ( 8 weeks post lesion), illustrating the tortuosity in CSA. Quantification of this tortuosity shows a time-dependent increased tortuosity in CSA. The orientations (caudal / rostral) apply to the three examples in B. Results are expressed as mean \pm SEM and are presented in overlay with individual vales. $N=5$ animals per group. Stats: ANOVA, followed by unpaired t-test as post-hoc test. * $\mathrm{p}<0.05, * *$ $p<0.01$. NS: Not statistically significant. ANOVA NS: ANOVA $p>0.05$. The orientations (caudal / rostral) apply to the three examples in A. ANOVA NS: ANOVA p>0.05. Bar : B: $1.3 \mathrm{~mm}$, D: $0.4 \mathrm{~mm}$.

\section{Figure 4}

See figure for legend. 

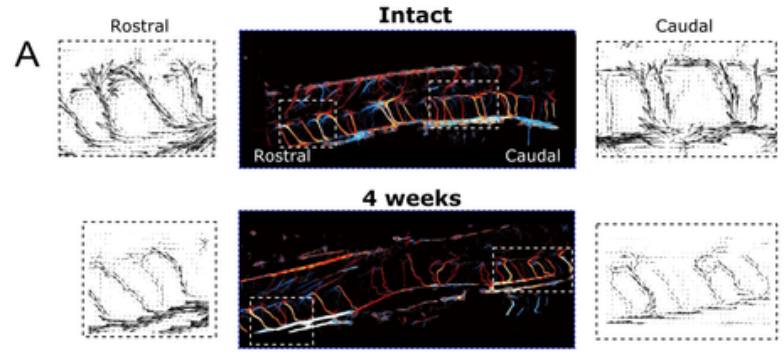

8 weeks
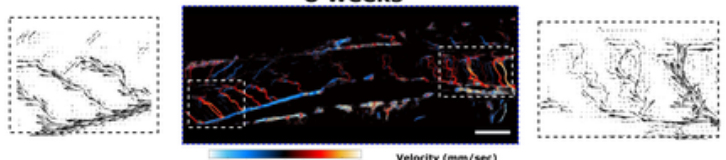

B
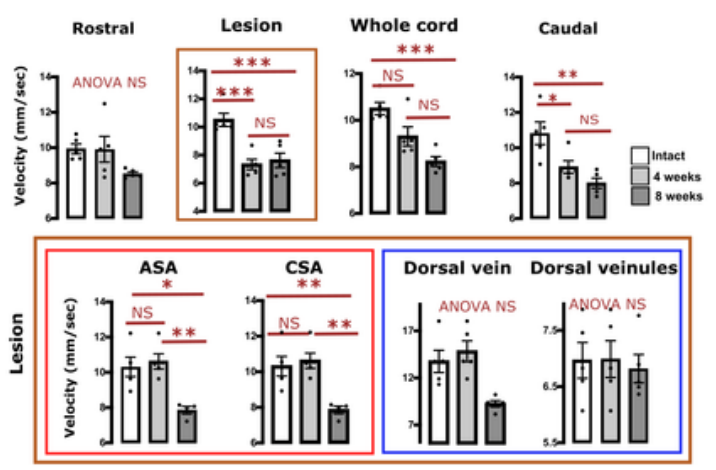

Figure 5: Quantification of bubble velocity using ULM reveals a timedependent reduction in the blood velocity caudal to, and within the lesion: in ASA and CSA. A: Representative examples of blood velocity (expressed in $\mathrm{mm} / \mathrm{sec}$, colored central panel) in animals of the different experimental groups: intact, 4 or 8 weeks post-contusion. Examples chosen are the same animals as in Figure 4. The lateral black and white panels illustrate field vectors of bubble trajectories (i.e. vectors illustrating the local propagation of the bubbles, using both horizontal and vertical velocities) in the rostral or caudal aspect of these examples. The size of the arrows is proportionate to the local speed. These areas are those delineated in white dashed rectangles in the colored panel. These examples illustrate the reduced velocity, associated with increased tortuosity of the vessels (quantified in figure 4 ; here visible at 8 weeks both on the rostral and caudal portions). B: Quantification of the bubble velocity in various vascular compartments. Results are expressed as mean \pm SEM and are presented in overlay with individual values. Stats: ANOVA, followed by unpaired t-test as post-hoc test. $* p<0.05, * * p<0.01, * * * p<0.001$. NS: Not statistically significant. ANOVA NS: ANOVA $p>0.05$. The orientations (caudal / rostral) apply to the three examples in A. Bar : $1.3 \mathrm{~mm}$ in the central coloured panels and $0.5 \mathrm{~mm}$ in the lateral black and white panels.

Figure 5

See figure for legend. 


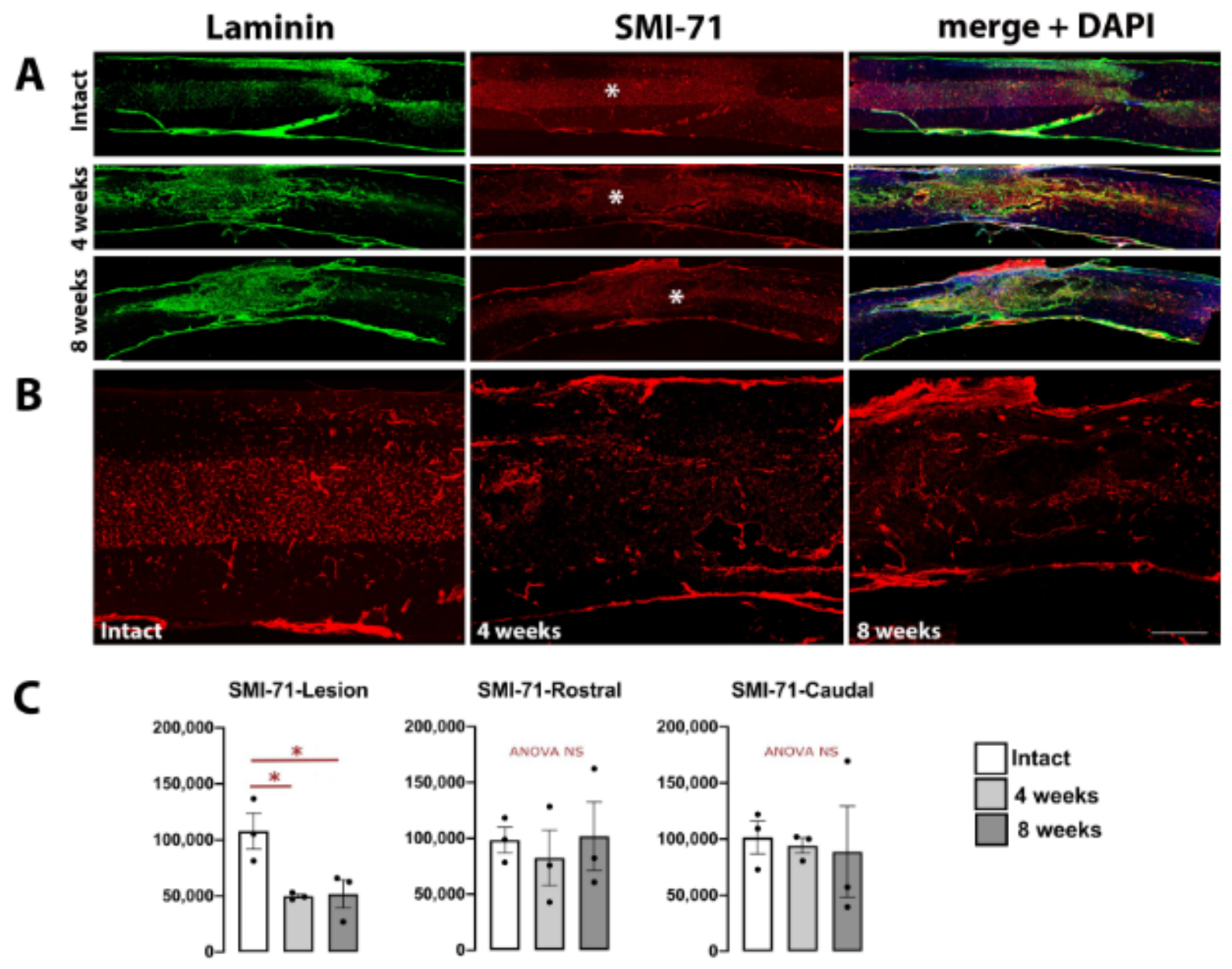

Figure 6 : A-Immunofluorescence labeling for laminin (green), SMI-71 (red), and DAPI (blue) on sagittal spinal cord sections from intact (non-injured) rat, and rats at 4- and 8-weeks post-contusion. B-Higher magnification of SMI-71 staining in A (stars) showing distribution and disruption of blood vessels in parenchymal contused spinal cord. Bar A: 12 mm, B: $300 \mu \mathrm{m}$.

Figure 6

See figure for legend. 

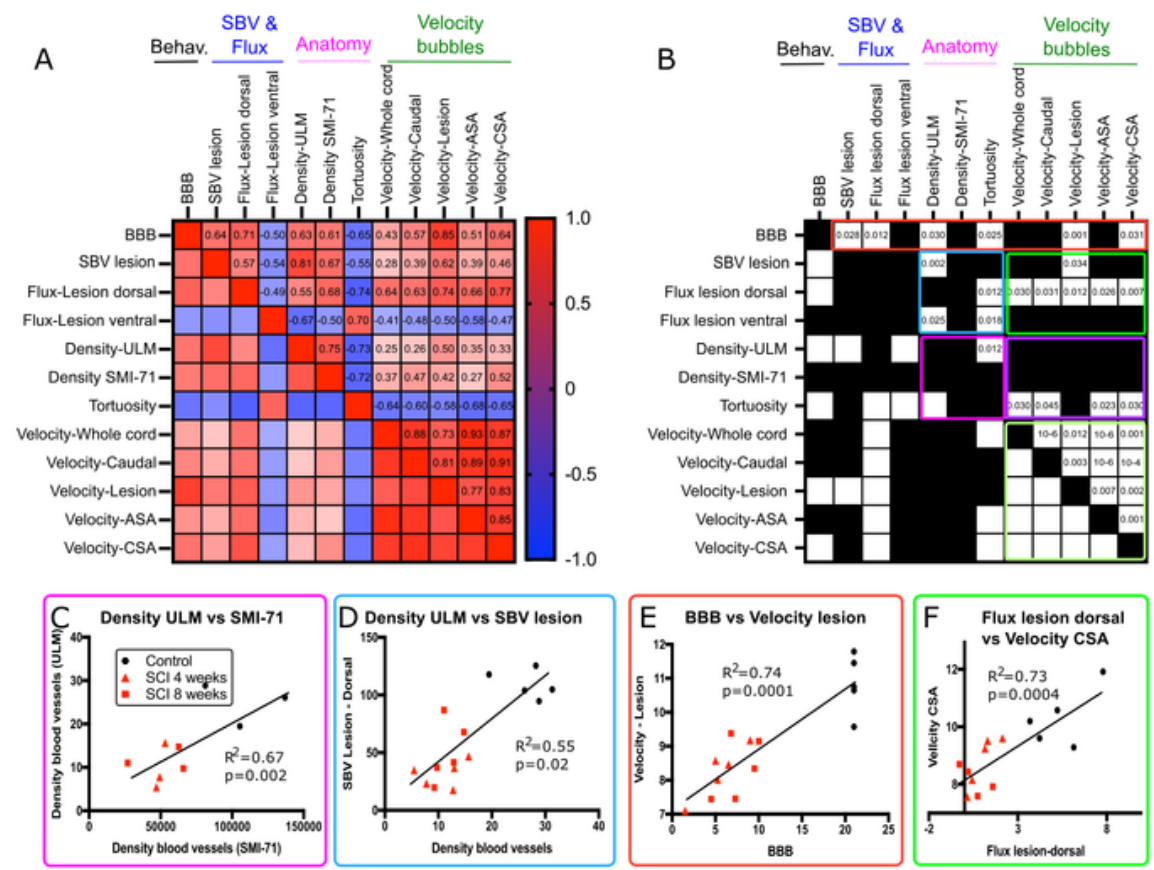

Figure 7: Statistically significant correlations between the locomotor disability of the animals (BBB), changes of SBV within the lesion, main flow, markers of altered vascular anatomy and altered velocity (ULM). Using individual results from all animals included in this study ( $\mathrm{N}=5$ per group), the putative correlation between these different outcome measures were determined using Spearman's correlation coefficient $(A)$, followed by calculation of the corrected $p$-value for multiple comparisons (B). In A and B, results are expressed as a double correlation matrix. In A: the color bar indicates the value of positive / negative correlations between these elements. In B: statistically significant cells (corrected $\mathrm{p}$ - value <0.05) are indicated in white. Black cells are statistically non-significant results. $A$ and $B$ : the individual values of either correlation $(A)$ or corrected $p$-value $(B)$ are given in the top half matrix. $A$ and $B, 8$ clusters of interest were highlighted: 1 ) red: correlation between the BBB and all functional and anatomical measures. 2) Green (bright and pale): correlation between the bubble velocity in various compartments or their correlation with the SBV/ flow. 4) Pink and violet: Correlation between various markers of the vascular integrity (measured both using fUS, ULM and immunohistochemistry) and bubble velocity. 5) Blue: Correlations between changes of SBV/flow and the vascular anatomical alterations. Values used for BBB are those of the week of imaging.

\section{Figure 7}

See figure for legend. 